\title{
Subject Index Vol. 56, 1995
}

Activated CD4+cells 516 Acute acalculous cholecystitis 389 - pancreatitis 502

Aging 230

Air enema X-ray films 528 Albumin 67 Alkaline tide 100 Allergy 421 ai-Antitrypsin 67

- deficiency 41

Aminopeptidase 400

Amoxicillin 14

Amylase 502

Anorectal motility 479

Anorectum 479

Antiulcer drugs 443

AntralGcell 31

AO-128 (voglibose) 493

Apical expulsion 175

Arginine 400

L-Arginine 463

Ascorbic acid 472

Autoimmune gastritis 455

Autoimmunity 41

Bacteria 159 Bacteroidaceae 415 Barrett's esophagus 347 Bifidobacterium 415 Bileacid(s) 165

- secretion 382

salt metabolism 523 Blood gas analysis 204 Body size 395

Bronchopulmonary lavage 204 Bullfrog 357

CaCo-2 cells (cultured intestinal cells) 57

Caerulein 127

Calcium-binding proteins 406

Carbachol 127,502

Cardiac innervation 488

Celiac axis compression syndrome 534

Cell interactions 335

- proliferation 364

B-Cell secretion 253

Ceruletid 389

Cholecystokinin octapeptide 502 Cholelithiasis 395 Cholestasis 429

Cholesterol 57

Choline-deficient, ethionine-supplemented diet 502

Cholyltaurine 165

Chondroitin sulfate 230

Chronic cholestatic liver disease 523 
hepatitis 199

pancreatitis 237 Cimetidine 509 Circadian rhythm 35 Cisapride 153 Clarithromycin 14 Clinical assessment 421 Colitis 370

Cold restraint 214

Colloid bismuth subcitrate 211

Colon 194

Colonic cancer 67

- $\quad$ polyp 67

Colonoscopy 528

Computer analysis 153

Controlled clinical trial 389

Crohn's disease 67, 377, 406

CyclosporinA 214

Cytokines 335

Cytoprotection 509

De-Nol 211 Dermatan sulfate 230 Dextran sulfate 259 Diabetes mellitus 493 Diagnostic procedures 76 Diet history 421 Dietary fibers 523 Differential diagnosis 76 Differentiation 57 Diffuse esophageal spasm 488 Dog 35 Duodenal tissue 230

ulcer $14,181,187$

- disease 25

- therapy, long-term 181 Dyspepsia 96,107

27E10 406

Eating physiology 253

Eicosapentaenoic acid 46

Electrorectogram 479

Electrorectography, transcutaneous 479

Endothelin 21,Ill,171

Enprostil 509

Epidemiology 377

Epidermal growth factor 364

Esophageal motility 153

peristalsis 153

reflux 347

Esophagus 488 Ethanol 145,220,509 European chameleon 76 Exocytosis 175 Experimental acute colitis 46

colitis 211,259

ulcerative colitis 159 Extracellular matrix 335 Extra-intestinal manifestations 76

Famotidine 145 Fat-storing cells 335 Fecal flora 415 Ferrous iron 472 FK506 259 Food

challenge 421

Gallbladder, emptying, volume, contour

395 Gastric acid secretion 220

acidity 35

blood flow 463

carcinoid tumour 455

cultured KATO III cells 364

damage 220, 357 


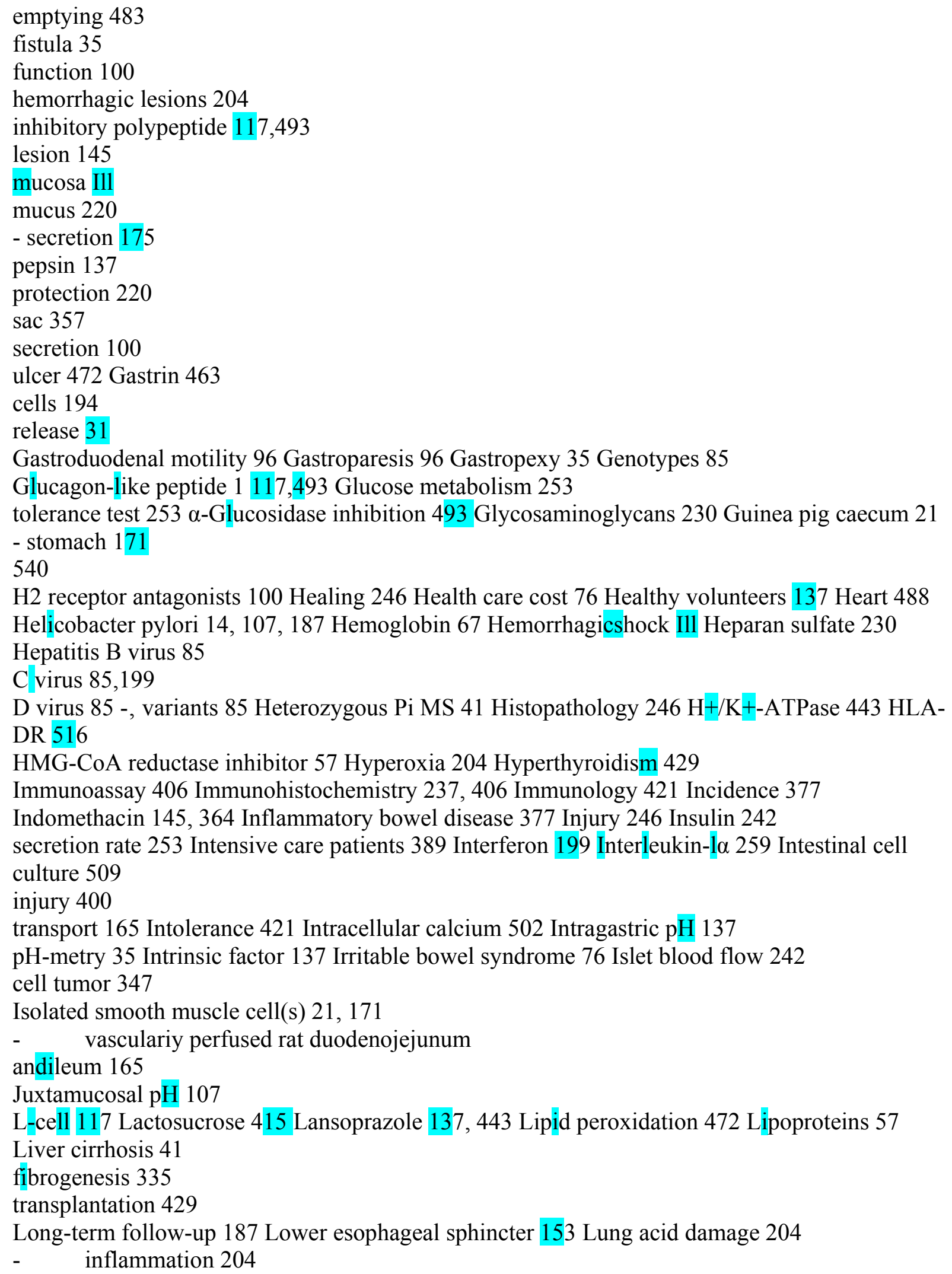


M3 muscarinic receptor 31 Macrophage 159 Mannitol 382 Manometry 96 Mastication 483 Meal stimulation 137 Medical treatment 199 (R)- $\alpha$-Methylhistamine 145 Mevinolin 57

Migrating motor complex 96 Misoprostol 220 Model 246 Motility studies 76 MRP 14406

MRP8 406 Mucin 52, 370 Mucosalcirculation Ill

injury Ill

lipid composition 46 Mucus 370

Mutants 85 Myeloperoxidase 214,259

Neostigmine 96

Nervous reflex 488

Nitric oxide 400,463

Nonsteroidal anti-inflammatory drugs 357 ,

364 Nutrient HCO5 357

Obesity 395

Omeprazole 14,25,181,194,220,443

- $\quad$ and amoxicillin combination 187

Ornithine 400

Oxygen radicals 472

Pancreas 127 Pancreatic blood flow 242

endocrine tumor 347

polypeptide 242,253 Pancreatitis 246 Pantoprazole 443 Pentagastrin 35 Perfused ileum 117

Phagocytosis 159

Phorbol myristate acetate 127 Phospholipase D 127 Polyamines 400,463 Polyethylene glycol

900382 Polymorphonuclear leukocytes 214 Prognosis 455

Progressive systemic sclerosis 230 Prokinetics 153

Proliferation 57 Prostaglandin(s) 357, 463

- $\quad$ E2 211

Proton pump inhibitors 443 Psychological factors 76 Psychosomatics 421

Quasi-species 85

Ranitidine 25, 96, 220 Rat 145,211,220,246 Receptor binding 364

- $\quad$ subtype 171

Rectum 479

Referrals 76

Reperfusion 400

Scanning electron microscopic observation

175 Serum gastrin 137

pepsinogen 137

soluble CD25 levels 516 Shark fin lipids 46 Sialylation 52

Smoking 370

Somatostatin cells 194

Stimulus-secretion coupling 502

Stools 67

Substituted benzimidazoles 443

Subtypes 455

Sucrase 400

Superoxide dismutase 472

Surgical implantation 35 
Symptom scoring 76

Systolic abdominal bruit 534

T cells 41

Teeth 483

Tetraprenylacetone 175

Thioperamide 145

Tissue culture 52

Transferrin 67

Transforming growth factor- $\beta \mathbf{i} 237$

Tumor necrosis factor- $\alpha 259$

Ulcer healing 463

Ulcerative colitis 52, 67, 377, 516, 528 Ultrasonography 395 Ursodeoxycholic acid 382, 523

Vagotomy 100

Vascular reconstruction 534

Voglibose(AO-128) 493

Zollinger-Ellison syndrome 347

541 